\title{
Augmented Reality to Improve Surgical Simulation: Lessons Learned Towards the Design of a Hybrid Laparoscopic Simulator for Cholecystectomy
}

\author{
Rosanna M. Viglialoro, Nicola Esposito, Sara Condino, Fabrizio Cutolo, Member, IEEE, Simone \\ Guadagni, Marco Gesi, Mauro Ferrari, and Vincenzo Ferrari, Member, IEEE
}

\footnotetext{
R. M. Viglialoro is with the EndoCAS Center, Department of Transla-tional Research and New Technologies in Medicine and Surgery, Univer- sity of Pisa, Pisa 56126, Italy (e-mail: rosanna.viglialoro@endocas.org).

N. Esposito, S. Condino, and F. Cutolo are with the EndoCAS Cen- ter, Department of Translational Research and New Technologies in Medicine and Surgery, University of Pisa.

S. Guadagni is with the Department of General Surgery Unit, Cisanello University Hospital AOUP.

M. Gesi is with the Department of Translational Research on New Technologies in Medicine and Surgery, University of Pisa and also with the Center Rehabilitative Medicine "Sport and Anatomy, University of Pisa.

M. Ferrari is with the EndoCAS Center, Department of Translational Research and New Technologies in Medicine and Surgery, University of Pisa and also with the Vascular Surgery Unit, Cisanello University Hospital AOUP.

V. Ferrari is with the EndoCAS Center, Department of Translational Research and New Technologies in Medicine and Surgery, University of Pisa and also with the Information Engineering Department, University of Pisa.
}

This work was supported by the Surgical Training in identification and isolation of tubular structures with hybrid Augmented Reality Simulation (SThARS) project(project code GR-2011-02347124) under the grant Young Researcher (under 40 years)/Giovani Ricercatori 2011-2012, by the Italian Minister of Health with the cofounding of Tuscany Region, the Virtual and Augu- mented Reality Support for Transcatheter Valve Implantation, by usingCardiovascular MRI (VIVIR) project (project code PE-2013-02357974) under the grant Ricerca Finalizzata, and by the Italian Minister of Health with the cofounding of Tuscany Region and the HORIZON 2020 Project VOSTARS, Project ID: 731974. Call: ICT-29-2016 Photonics KET 2016.

(Corresponding author: Rosanna M. Viglialoro.)

Index Terms - Surgical simulator, laparoscopic simulation, augmented reality, hybrid simulators, physical anatomical model, cholecystectomy training.

\begin{abstract}
Hybrid surgical simulators based on augmented reality (AR) solutions benefit from the advantages of both the box trainers and the virtual reality simulators. This paper reports on the results of a long development stage of a hybrid simulator for laparoscopic cholecystectomy that integrates real and the virtual components. We first outline the specifications of the AR simulator and then we explain the strategy adopted for implementing it based on a careful selection of its simulated anatomical components, and characterized by a real-time tracking of both a target anatomy and of the laparoscope. The former is tracked by means of an electromagnetic field generator, while the latter requires an additional camera for video tracking. The new system was evaluated in terms of AR visualization accuracy, realism, and hardware robustness. Obtained results show that the accuracy of AR visualization is adequate for training purposes. The qualitative evaluation confirms the robust- ness and the realism of the simulator. In conclusion, the proposed AR simulator satisfies all the initial specifications in terms of anatomical appearance, modularity, reusability, minimization of spare parts cost, and ability to record surgical errors and to track in real-time the Calot's triangle and the laparoscope. Thus, the proposed system could be an effective training tool for learning the task of identification and isolation of Calot's triangle in laparoscopic cholecystectomy. Moreover, the presented strategy could be applied to simulate other surgical procedures involving the task of identification and isolation of generic tubular structures, such as blood vessels, biliary tree, and nerves, which are not directly visible.
\end{abstract}




\section{Introduction}

Several medical simulators exist today on the market mainly due to the increasing demand for minimally invasive surgical (MIS) procedures and the increasing concern on patient safety [1]. Only 2 years ago the front cover of IEEE Pulse was dedicated to medical simulation with the subtitle "New Tech Reconfigures the Training Landscapes" [2].

Current MIS simulators are mainly implemented with two opposed approaches: virtual reality (VR) simulators, and physical simulators; each category comes with its own advantages and disadvantages [3].

VR simulators allow the repetitive execution of basic tasks (cutting, grasping, suturing etc.) and of the whole surgical procedures (such as laparoscopic and endoscopic procedures e.g., cholecystectomy), and they provide precise metrics for the evaluation of the trainee performance [4]. Such simulators range from serious gaming applications which are becoming more widespread to complex simulation platform [5], [6]. For example, Touch Surgery (TS) (Kinosis Limited, London, UK), is a serious gaming cost-free application for cognitive task simulation and rehearsal of key surgical steps [7].

On the other hand, an example of complex simulation platform is represented by LapMentor (Simbionix, USA). This is a trainer for laparoscopic surgery available in two versions with and without force feedback. Both versions have many modules including fundamental laparoscopic skills and full laparoscopic procedures (e.g., cholecystectomy, etc.). Numerous studies have demonstrated its validity. However, the complex simulation plat- forms require ongoing technical support and system upgrades and they have the costs very high making it prohibitively expensive for many institutions [8]-[10].

Overall, a limitation of VR simulators is the unrealistic simulation of the visual and haptic sensations between virtual objects and instruments [8].

The physical simulators are used for the acquisition of fundamental skills, such as hand-eye coordination, perception of depth of field, and manual skills needed for performing specific surgical tasks comprising the use of real instruments (e.g., suturing, dissection etc.) [11]. Such simulators range from simple box trainers to human torso models with simple objects such as pegs and inanimate models of human organs.

One important advantage of the physical simulators is the natural haptic feedback during the task execution. Besides, their costs are relatively low (a few thousand dollars) [9]. However, major disadvantages of this second approach are: the lack of an automatic evaluation of the user's performance, its non- reusability in case of destructive tasks, and the difficulty in providing highly detailed and anthropomorphic replicas of the organs.

A relatively innovative approach is the use of AR technology to implement hybrid simulators [12][16]. According to the physical-virtual simulation spectrum proposed by Samsun Lampotang et al. [17], AR simulation is a form of mixed simulation which combines physical simulation (such as laparoscopic equipment or/and mannequins) and VR simulation within a unique simulation environment.

AR simulators retain the natural haptic feedback of the physical simulation and the performance evaluation tools typical of VR simulation [12].

Barsom et al. [18] presented a systematic review on AR simulators for medical training. The authors divided the AR simulators into three categories on the bases of training purposes: neurosurgical procedures, echocardiography and laparoscopic surgery.

We focused our attention on the last category. Specifically, existing AR surgical simulators for MIS training are very few both at commercial and research level. The only commercial AR surgical simulator is the ProMIS (Haptica, Dublin, Ireland). Today its production is temporarily stopped because the system was acquired by CAE Healthcare. The system offers the simulation of basic skills and some laparoscopic procedures (e.g., appendectomy, colectomy, cholecystectomy). The system com- bines a laparoscopic mannequin connected to a laptop and a virtual environment. It 
uses real surgical instruments which are tracked during the tasks to provide an accurate and objective assessment of the user's performance. Different physical models such as suturing pads, can be inserted into the mannequin.

However, the included physical models are neither highly detailed nor anthropomorphic. In addition, the simulator does not allow either to update virtual anatomy following deformations impressed on physical anatomical models or to add simulated physiological functionalities. Indeed, virtual information is applied to complete the surgical scene with organs not included in the synthetic environment, and to provide visual instructions and guidance information: for example, it indicates where to cut a tissue and it shows the "virtual jet" of blood in case of tissue damages [8].

To date, ProMIS is the most validated surgical simulator and, as already said, the only commercial system featuring AR functionalities [12], [13], [19].

Concerning the non-commercial AR laparoscopic simulators, Lahanas et al. have described an AR simulator for MIS basic skills (e.g., navigation, peg transfer and clipping). The system allows the real-time tracking of the laparoscopic instruments posed inside a box trainer and the interaction with various virtual elements rendered on the screen. The authors provide an evidence for face and construct validity [8], [20].

However, existing AR laparoscopic simulators have a limited AR experience and they allow only the execution of simple tasks. In addition, they are often based on physical models that are not highly detailed, non-anthropomorphic.

To the best of our knowledge, we believe that so far, the real potentialities offered by AR technologies have not been explored yet in the context of surgical simulation.

Accordingly, we explored the potentialities of AR in surgical simulation for the training of identification and isolation of deformable tubular structures in surgery [21], [22].

In [21], we described an AR proof-of-concept solution that allows the AR visualization of hidden tubular structures sensorized with miniature electromagnetic (EM) sensor coils. In the same paper, we also motivated why the use of EM tracking is the best technology available nowadays. Furthermore, in [22], we applied the proposed AR solution to visualize and to recognize different configurations of the Calot's triangle in simulated cholecystectomy procedures. In all these works the augmented scene was generated by combining the image captured by a fixed camera and the information from the EM tracking.

In this paper, we report on the lessons learned during the development of an AR advanced simulation platform for laparoscopic cholecystectomy (LC), and we show the results of its evaluation. LC is the standard of care for gallbladder removal and the most commonly performed laparoscopic surgical procedure [23].

However, a serious and devastating technical complication of the LC is bile duct injury (BDI) [24]. There are mainly two factors increasing the BDI risk: the misinterpretation of the anatomy, mentioned by $92.9 \%$ of surgeons as the primary factor, and the lack of experience in LC, mentioned by $70.9 \%$ of surgeons as a contributing factor [25]. Indeed, although laparoscopic surgery (LS) is beneficial to the patient (small incisions, short hospital stays, minimal postoperative pain, etc.), it is technically more demanding and stressful than the traditional surgery, and it requires greater concentration [26].

General complications of LS are often related to the commonly known drawbacks of the laparoscopic environment: the two-dimensional images provided by the laparoscope do not allow a clear-cut vision as in the open intervention, therefore the surgeon significantly loses the perception of depth; the instruments are difficult to manipulate due to the fulcrum effect; the use of minimally invasive surgical tools causes the loss of tactile sensation [27]-[29]. To prevent avoidable surgical complications and to fully master the procedure, the surgeon must acquire the specific technical skills which can be achieved through appropriate training in LS. For this purpose, the simulation offers a safe and practical way to gain and to assess skills, through repeated practice, outside the operating theatre [30]. 
As already mentioned, in our previous studies [21], [22], [31], we presented a proof-of-concept of AR simulation platform for LC to practice identification and isolation of the Calot's triangle. Based on our previous works, this paper presents the final design specifications of the AR simulator taking into account the iterative feedback obtained by surgeons who have tested our previous demonstrators. In detail, we described an innovative simulation strategy which combines highly detailed physical models and virtual reality information in a surgical scene. The strategy deeply integrates both visual AR and acoustic functionalities, improving the state of the art. In comparison to our proof-of -concept system, the current system includes the localization of laparoscope. Specifically, we present and implement a solution for calibration that involves an additional camera for video tracking. The localization of laparoscope allows its free movement maintaining the geometric coherence of the AR scene. The quantitative tests are aimed to evaluate the AR visualization accuracy. In addition, not only the usefulness of AR visualization but also the experimental results for the evaluation of the complete system in terms of both robustness and realism of the physical components have been reported for the first time, in this paper.

Finally, this manuscript provides manufacturing details for the implementation of the simulator. The paper is organized as follows:

- An overview about design specifications and a detailed description of the physical components of the simulator.

- The methodologies for AR implementation and laparoscope tracking and the results of the experimental tests. The tests are aimed to evaluate the AR visualization accuracy and qualitatively verify the simulator robustness and realism.

- A thorough discussion about all the issues addressed during the development of the simulator and possible future improvements and further fields of applications.

\section{Material and Methods}

\section{A. Design Specifications}

The basic design specifications for our hybrid LC simulator are: realistic anatomical appearance, modularity, reusability, minimization of spare parts cost, AR visualization, ability to signal surgical errors and to track in real time both Calot' s triangle structures and laparoscope. Here follows a detailed description of the specifications:

1) Realistic anatomical appearance: The simulated anatomy is chosen taking into account all the anatomical structures that could be either seen or touched during the execution of the procedure.

The anatomical replicas should match morphology, topology, color, texture, density and they should mimic the mechanical behavior of the real structures as far as possible. Therefore, our simulator includes the following replicas: liver, gallbladder, biliary tree, arterial tree, pancreas, abdominal aorta, esophagus- stomach-duodenum and connective tissue.

2) Modularity: Due to the morphological and topological variations that occur naturally in human hepatobiliary anatomy, the prototype is designed to be modular, so that anatomical parts such as connective tissue, biliary tree (BT) and arterial tree (AT) can be easily and separately replaced. In particular, BT and AT, which are sensorized and thus expensive, are designed to be reusable. In our sim- ulator, different anatomical variations are predisposed, and an easy connection/disconnection coupling is imple- mented to allow any easy substitutions on demand, for training sessions with different complexity. The connec- tive tissue, fabricated with a low-cost material, is instead disposable.

3) Reusability and minimization of spare parts cost: Reusability and minimization of spare parts cost are closely linked. All the anatomical structures, except from the connective tissue which must be dissected, are to be fabricated using materials that are reusable and extremely durable over 
time. For this reason, silicone and nitinol tubes are used in order that the overall training costs are reduced.

4) AR visualization: The simulator offers an AR visualiza- tion of the hidden tubular structures (AT and BT). AR visualization has twofold function: it provides the trainee information about the implemented anatomical variations and it provides a way to verify the correct isolation of the target structures after the trial.

5) Signaling surgical errors: The prototype is designed to enable acoustic signaling of potential damages to the BT and/or AT walls. In this way, the simulator can directly evidence a lack of surgical skills and provide a way to evaluate the performance.

6) EM tracking of the Calot's triangle structures: The proto- type is designed to enable the EM tracking of the Calot's triangle structures.

7) Localization of the laparoscope: The prototype is de- signed to enable the real-time tracking of the laparoscope. In this way, it is possible to freely move the laparoscope maintaining the geometric coherence of the AR scene. This aspect is fundamental during laparoscopic proce- dures. To this end, the strategy requires an additional camera for optical detection and tracking of a structured marker.

In the following sections, we outline the steps that led to the development of the final simulator, from the Computer Aided Design (CAD) project to its fabrication, and then we describe a preliminary evaluation of the simulator. Sections are:

B) CAD Project of the real simulator components

C) Fabrication of the real simulator components

D) Overview of AR simulator

E) Evaluation of AR simulator

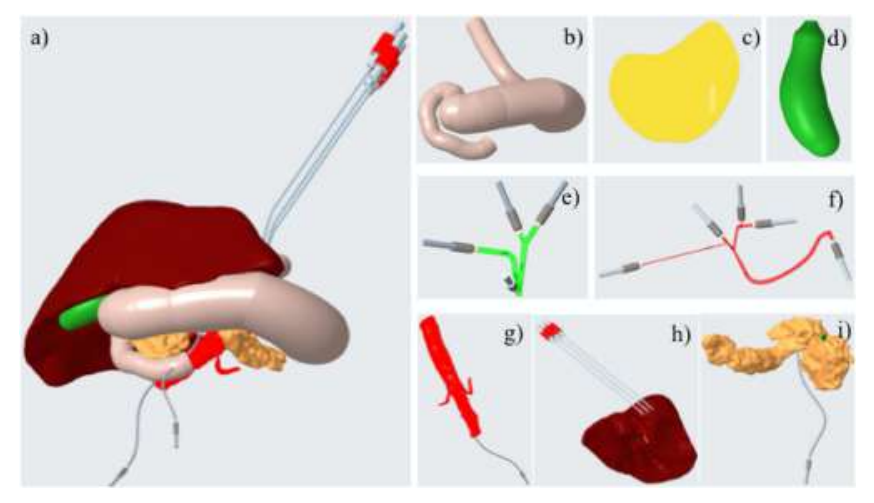

Fig. 1. CAD project of the simulator real components: a) $3 D$ model of whole simulator; b-i) 3D model of each component of simulator: b) Esophagus-Stomach-Duodenum; c) Connective tissue, d) Gallbladder;

e) Biliary tree; f) Arterial tree; g) Aorta; h) Liver; i) Pancreas

B. CAD Project of the Real Simulator Components

The first step involves the CAD project of the whole real simulator components by means of the PTC Creo Parametric

2.0 software. The CAD project integrates both $3 \mathrm{D}$ anatomical models and $3 \mathrm{D}$ models of the required electronic accessories (Fig. 1).

C. Fabrication of the Real Simulator Components

1) Simulator Components: Based on the adopted fabrica- tion strategy [21], [22], [31], [32], the anatomical real compo- nents of the simulator can be divided in two categories: 
. Patient specific models: liver, gallbladder, pancreas, ab-dominal aorta, esophagus-stomachduodenum.

. Non-patient specific anatomical models designed in the CAD environment: AT, BT and connective tissue.

Patient specific models are obtained starting from the CT im- ages of a real patient, selected by the surgeons as gold standard patient in terms of morphology and volume of the organs involved. Nonpatient specific anatomical models are added to the gold standard anatomy to simulate anatomical variations.

The strategy used for the patient specific models' fabrication consists of four 4 main steps: extraction of the 3D models of the target organs starting from CT images; molds designing in the 3D CAD software; molds manufacturing with a 3D printer (Dimension Elite 3D Printer); casting of the chosen materials into the molds.

As for the first step, models are segmented by means of the EndoCAS pipeline developed in our center on the top of the open source software ITKSNAP [32], [33].

Casting materials are mostly silicone rubbers (Smooth-On Inc, Easton PA) often mixed with some additives in order to mimic tissues properties [32], [33].

The Table I shows the selected mixtures for the fabrication of each patient specific models. The Fig. 2 shows all the anatomical replicas of the simulator.

The manufacturing process of the AT and BT involves the use of nitinol tubes, with external and internal diameters of

$0.89 \mathrm{~mm}$ and $0.53 \mathrm{~mm}$ respectively, joined together by tin wires and covered by a thin silicone layer. This allows the fabrication of flexible structures with diameters comparable to the natural ones. In detail, the synthetic model of arterial tree includes the cystic artery (CA), left and right hepatic arteries (LHA and RHA), and proper hepatic arteries (PHA). The model of biliary tree includes the cystic duct (CY), right and left hepatic ducts (RHD and LHD), common hepatic duct (CHD) and choledochus duct (CD).

The number of nitinol tubes varies according to the size of the portion of AT and BT and to the anatomical variants that we want to reproduce. At present, two anatomical variants are designed both for $\mathrm{CA}$ and $\mathrm{CY}$. This allows the simulation of four different anatomical configurations frequently encountered during the cholecystectomy.

Table II shows the chosen anatomical variants of CA and of CY and the number of nitinol tubes used for the fabrication of each portion of AT and BT.

The manufacturing process takes into account the need of:

. sensorizing the Calot's triangle for the implementation of the AR functionalities;

. using conductive materials for the detection of electric contact between BT and/or AT and the surgical instru- ments by means of sound warnings.

As already described in [21], commercially available 5 de- grees of freedom (DOF) EM sensor coils (0.50 mm diameter, $8 \mathrm{~mm}$ length) are used to implement an AR solution allowing the real-time visualization of the Calot's triangle. In detail, three sensors are used for CA and four sensors for CY. The sensors' placement was empirically chosen to optimize the virtual re-construction of the Calot's Triangle configuration (see the "AR Visualization" paragraph), which is an indisputable anatomical landmark of cholecystectomy [34]. Fig. 3 shows the position of EM sensors inside each anatomical variants of $\mathrm{CA}$ and of $\mathrm{CY}$.

Finally, the strategy adopted for the fabrication of the con- nective tissue, which has to be dissected during the simulation and then replaced for each trail, involves the use of a degradable material. A gelatinous material, made of Psyllogel Fiber pow- der, is adopted to simulate the consistency of the natural tissue. The synthetic tissue is produced in the form of thin sheets, as shown in Fig. 4, and is attached to the other anatomical parts by adhesive velcro strips. 


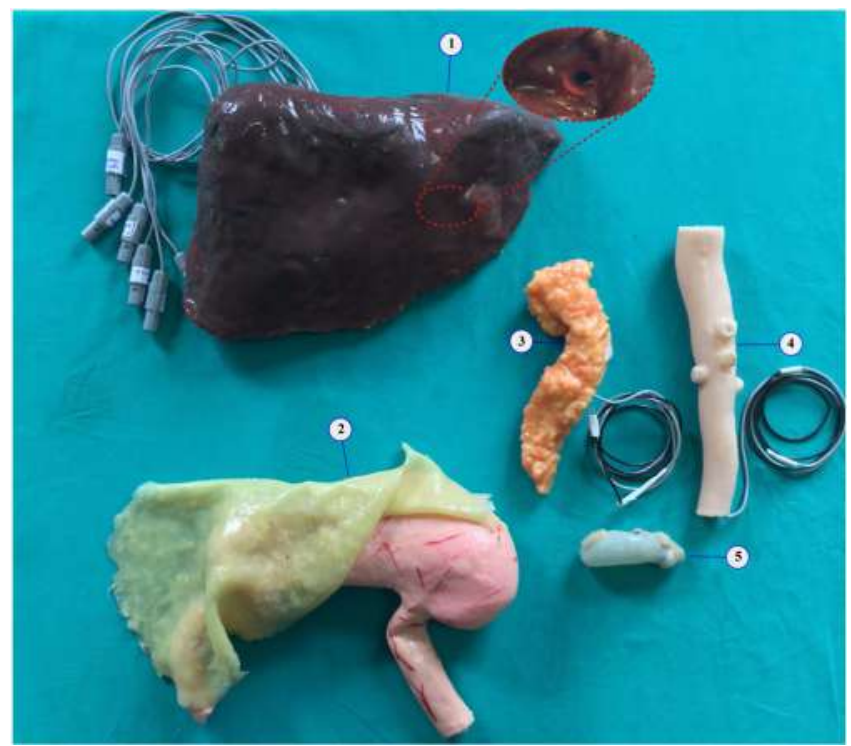

Fig. 2. Anatomical replicas: Liver, Esophagus-Stomach- Duodenum, Pancreas, Aorta; Gallbladder; The zoomed detail shows the female connector inside the liver

TABLE I

Selection of Mixtures for Realization of Anatomical Replicas

\begin{tabular}{|c|c|c|c|c|c|c|c|}
\hline $\begin{array}{l}\text { Specific-patient } \\
\text { replica }\end{array}$ & ECOFLEX 0010 & $\begin{array}{c}\text { Dragon Skin- } \\
\text { Fx-Pro }\end{array}$ & Slacker & Thinner & Vaseline oil & Natural fibers & $\begin{array}{c}\text { Power } \\
\text { Graphite }\end{array}$ \\
\hline Liver & $\mathrm{X}$ & $\mathrm{X}$ & $\mathrm{X}$ & $\mathrm{X}$ & $\mathrm{X}$ & - & $\mathrm{X}$ \\
\hline Gallbladder & - & $\mathrm{X}$ & - & - & - & $\mathrm{X}$ & - \\
\hline $\begin{array}{l}\text { Abdominal } \\
\text { aorta }\end{array}$ & - & $\mathrm{x}$ & - & - & - & - & - \\
\hline Pancreas & $\mathrm{x}$ & $\mathrm{x}$ & - & - & - & - & - \\
\hline $\begin{array}{l}\text { Esophagus- } \\
\text { Stomach- } \\
\text { Duodenum }\end{array}$ & - & & - & - & - & - & - \\
\hline
\end{tabular}




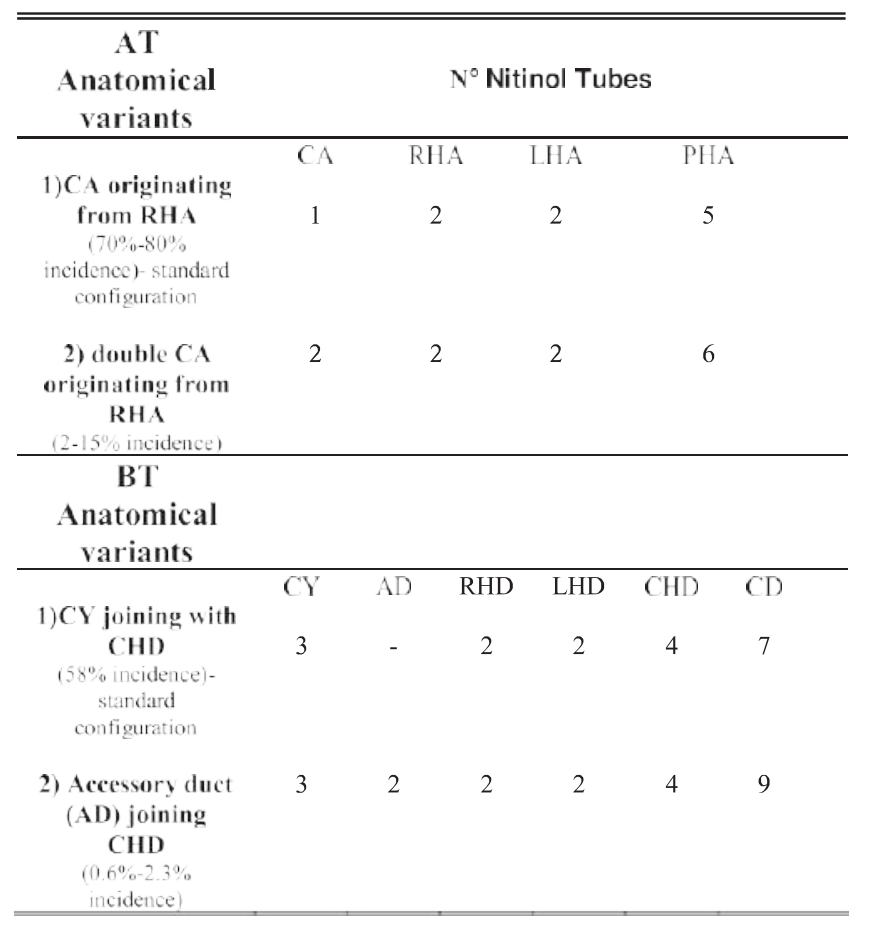

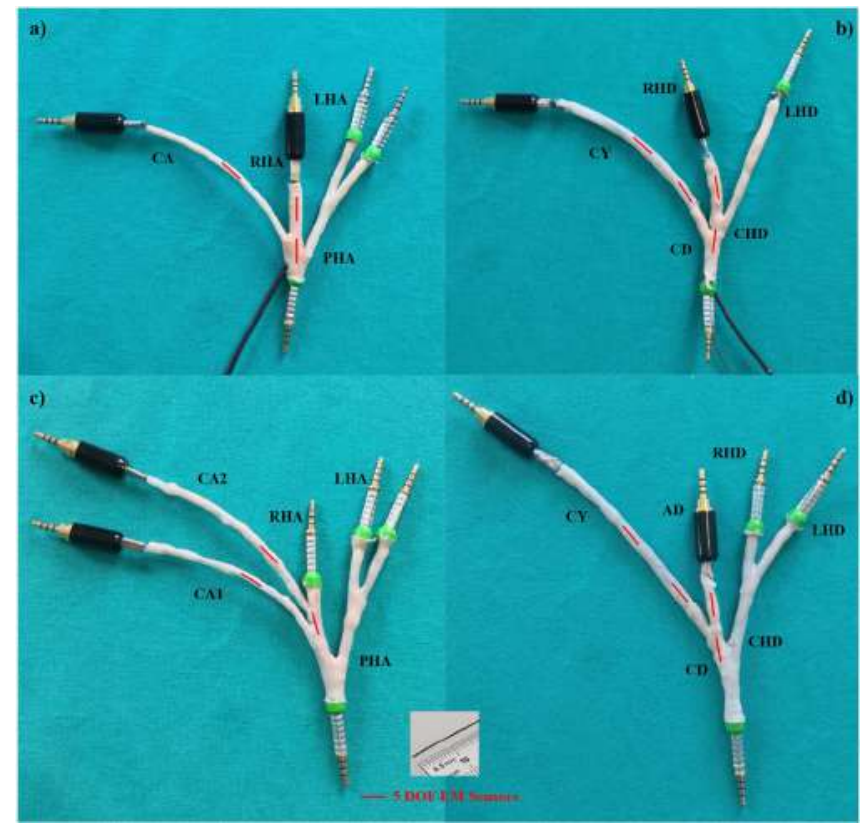

Fig. 3. Anatomical variants of AT and BT: a) CA originating from RHA (standard configuration); b) $\mathrm{CY}$ joining with CHD (standard configura- tion); c) double CA originating from RHA; d) accessory duct (AD) joining CHD. The red cylinders represent the sensor' position.

2) Method of Connection between the Anatomical Com- ponents: A fundamental aspect in the development of the sim- ulator, in addition to its realism, is represented by the method- ology adopted to link the different anatomical components. All the links, except from one, are made through connectors for the purpose of facilitating the anatomical parts replacement (as BT and AT) and of ensuring the simulator modularity. Only the connective tissue is attached to the gallbladder 
by means of ad- hesive velcro strips. To better understand the chosen connection strategy, we report below a brief description of the anatomical relationships between AT and BT and the adjacent organs:

. anatomical relationships of BT with the liver, gallblad-

der and pancreas: The BT begins with RHD and LHD emerging from liver and coming together in the CHD. The latter connects to the CY to form the CD that passes through the pancreas head and opens into the duodenum. Finally, the cystic duct joins the gallbladder at the level of CHD. The accessory or aberrant ducts typically emerge from the right lobe of the liver.

. anatomical relationships of AT with the liver, gallbladder and aorta: The PHA arises from the common hepatic artery (CHA) that arises from the celiac trunk; and then it terminates by bifurcating into the RHA and LHA before entering the porta hepatitis of the liver. Finally, the CA that enters the gallbladder in the neck or body area originates from the RHA.

Therefore, in order to respect the anatomical relationships among the anatomical components, pairs of matching $2.5 \mathrm{~mm} 4$ poles jack connectors (female-male) are inserted at the entry points ends of AT-BT branches. In detail, the upper and lower ends of the BT and AT are equipped with a male connector whose function can be either purely mechanical or mechanical- electrical. The male mechanical-electrical connectors are posi- tioned at the upper ends of the sensorized branches (max 2 sen- sors for each connector since each sensor is a coil with 2 wires) and at the lower ends of the $\mathrm{CD}$ and PHA [22]. In addition, the nitinol tubes containing the sensors wires, are crimped with the connector itself to guarantee enough mechanical robustness at the interface between the thin sensor wires and connector.

The Table III shows, for every anatomical variant of AT and BT, the classification of male connectors on the bases of their functions.

The corresponding female wired connectors, enclosed in an ad-hoc designed box, were embedded into the liver, the pancreas and the aorta replicas respecting the anatomical relationships among the AT/ BT and adjacent organs. In addition, each fe- male connector (with mechanical-electric function) embedded into the liver replica, terminates in a tool connector (outside the liver replica) for the interconnection between the LC simulator and the EM Tracking system. The Fig. 5 illustrates the assem- bly and the exploded view of the simulator 3D models. The latter view shows the location of mechanical and mechanical- electrical connectors inside each component. 


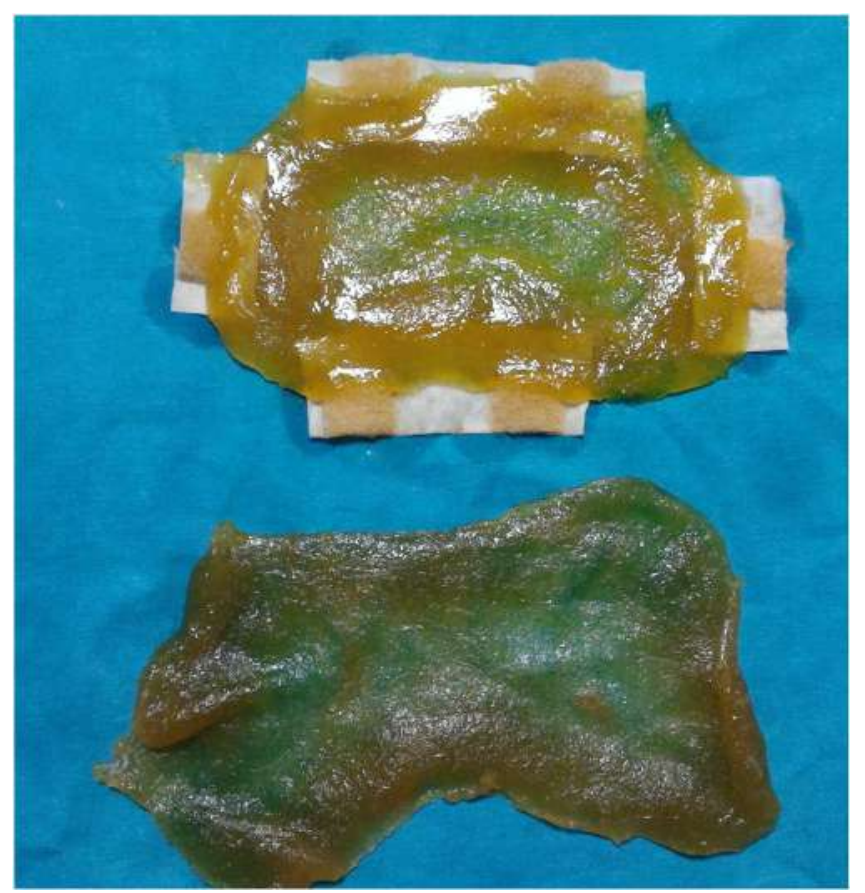

Fig. 4. Synthetic connective tissue.

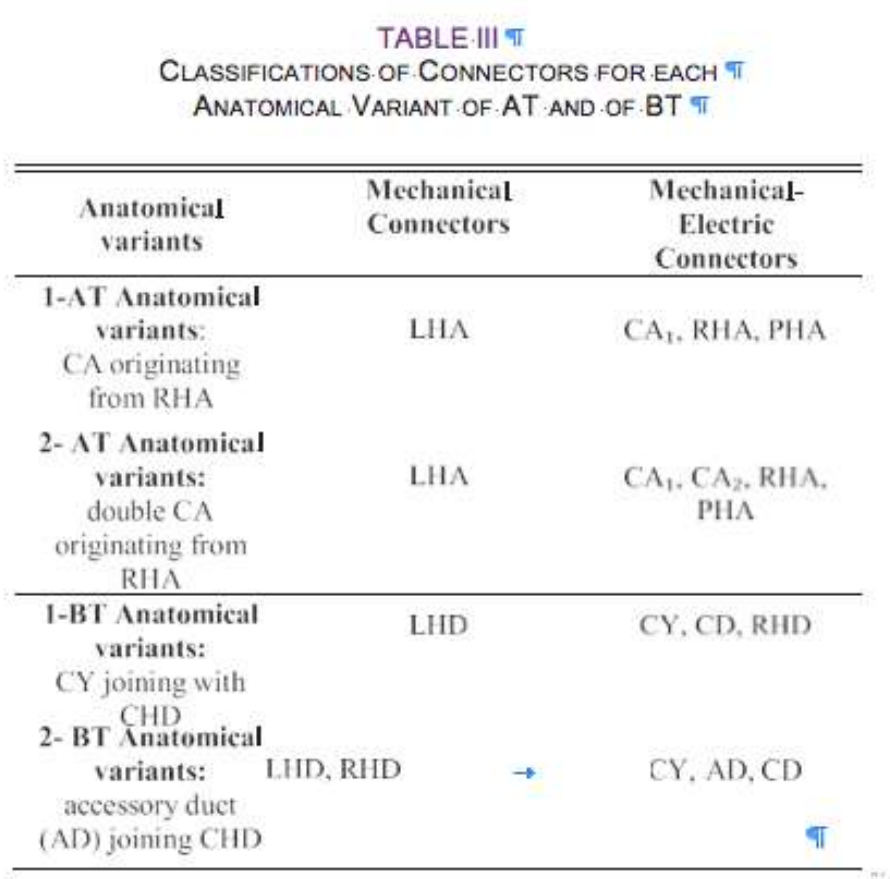

3) Acoustic Functionality: A simple electric circuit is cre- ated to signal eventual damages to the BT and/ or AT walls. The BT, the AT and a surgical hook are electrically connected to an electric circuit in a way that the surgical hook contact with the conductive structural frames of BT or AT causes the closing of the circuit [31]. The latter is signaled both with a sound alarm generated by a buzzer and with the illumination of a red or yel- low led depending on the touched structure. In this way, it is possible to distinguish if the damaged structure is the AT or the BT. Fig. 6 shows the electric circuit and the equivalent electric scheme. 


\section{Overview of the AR simulator}

The system is set up to track in real time both the laparoscope and the Calot's triangle sensorized real components and to co- herently visualize the virtual content on the real laparoscopic image (Fig. 8).

In this section, the system set-up, the calibration procedure, and the AR visualization are described in detail.

1) The System Set-up: The set-up consists of the follow- ing components: a laparoscope, a commercial box trainer, the real physical simulator components, two structured markers for optical tracking, an EM tracking system (Aurora, Northern Dig- ital Inc., Waterloo, ON, Canada), a USB camera and a laptop PC.

The employed laparoscope comprises the following two main components: an image sensor with a resolution of 1024768 pixels and a forward-oblique $30^{\circ}$ HOPKINS II telescope (KARL STORZ TELEPAK 20043020 UNIT by Karl Storz En-

doskope $\mathrm{GmbH}$ and Co. KG, Tuttlingen, Germany). The latter is commonly used for abdominal surgery.

The USB camera is rigidly mounted perpendicular to the la- paroscope shaft, and it provides the position and orientation of the laparoscope (as detailed in the following sections). The USB 2.0 camera (uEye XS by IDS Imaging Development Sys- tems GmbH, Obersulm, Germany) has a resolution of 1280720 pixels. Since in such laparoscopes it is possible to rotate the optics with respect to the camera head in order to change the image "horizon", we 3D printed an ABS block to block this degree of freedom. This block was mounted between the laparoscope shaft and the camera head of the laparoscope, (as shown in Fig. 7); in this way, we avoided having to re-estimate the camera-to-laparoscope pose each time the shaft was rotated.

Two structured markers (e.g., checkerboard), respectively Camera Grid (CG) and the Laparoscope Grid (LG), arranged as depicted in Fig. 8, are used for both calibration purposes and the localization of the laparoscope as explained in the following paragraph.

The physical simulator and the two calibration grids are in- serted in a commercial box trainer (Intuitive Surgical, Inc.).

The Aurora Tabletop EM Field Generator, employed for the tracking of the BT and AT, is placed under the box trainer. Finally, a laptop PC (UX303UB by ASUS, provided with an Intel Core i56200U CPU@2.30 GHz core processor, an 8 GB RAM and with a graphics card NVIDIA GeForce $940 \mathrm{M}$ ) is used to run the AR visualization software.

2) Cameras Intrinsic and Extrinsic Parameters Calibra- tion: An accurate calibration of the intrinsic (projective) and extrinsic (pose) parameters of the laparoscope and of the USB camera is essential for accurate AR visualization. For both video cameras, the intrinsic parameters are estimated using the Zhang's method (as implemented in Matlab toolbox for camera calibration) [35] acquiring the dedicated calibration grid (the CG and the LG respectively) from 20 various positions. The obtained root mean square (RMS) error of the calibration procedure was of 0.18 and 0.2 pixels for the laparoscope and camera respectively, which indicates a good calibration accu- racy for the intrinsic parameters. For computing the extrinsic parameters, we considered the electromagnetic field generator as the global reference frame. Fig. 8 shows all the involved coordinate frames and transformations.

The laparoscope extrinsic parameters describe, in our case, the geometric relationship between the laparoscope coordinate frame L, and the electromagnetic field generator E. The corre- sponding transformation is denoted by LTE $(\mathrm{t})$, which changes over time as a function of the current position of the laparoscope:

${ }^{\mathrm{L}} \mathrm{TE}(\mathrm{t})={ }^{\mathrm{L}} \mathrm{TC} *{ }^{\mathrm{C}} \mathrm{TCG}(\mathrm{t}) *{ }^{\mathrm{CG}} \mathrm{TLG} *{ }^{\mathrm{LG}} \mathrm{TE}$

Where:

${ }^{\mathrm{L}} \mathrm{TC}$ is the static homogeneous transformation between the coordinate frame L and the USB camera coordinate frame $\mathrm{C}$; 
${ }^{\mathrm{C}} \mathrm{T}$ CG $(\mathrm{t})$ is the homogeneous transformation, at the instant $\mathrm{t}$, between the coordinate frame of $\mathrm{C}$ and of CG; Fig 8: transformation (represented by dashed line) and Coordinate frames;

CG TLG is the static homogeneous transformation between the coordinate frame CG and the LG;

LG TE is the static homogeneous transformation between the coordinate frame LG and the E.

${ }^{\mathrm{L}} \mathrm{TC}$ is calculated offline with the hand-eye calibration

method.

This method is widely used in robotics [36].

This calibration problem yields a homogeneous matrix equa- tion of the form:

$\mathrm{AX}=\mathrm{XB}$

In our case, the robot's hand and eye are respectively replaced by the laparoscope and the USB camera.

Fig. 9 shows the transformation matrix involved in the calibra- tion process, which requires to move the laparoscope in several positions denoted by the index i. For simplicity, the original notation of (2) is maintained in the following description of the calibration process:

. $\mathrm{X}$ is the unknown homogeneous transformations between $\mathrm{C}$ and $\mathrm{L}$ coordinate frames.

. A is the homogeneous transformation that describes the relative motion between two consecutive poses of $\mathrm{C}$ in the $\mathrm{CG}$ relative system.

. $\mathrm{B}$ is the homogeneous transformation that describes the relative motion between two consecutive poses of $\mathrm{L}$ in the $\mathrm{LG}$ relative system.

$\mathrm{X}$ is estimated using hand-eye calibration method by Park [36]. The laparoscope and the USB camera are moved in 20 different positions; for each pair of poses " $i$ ", the relative motion of each coordinate frame $(\mathrm{C}$ and $\mathrm{L})$ is recorded and stored in the two homogeneous transformation matrices: $\mathrm{Ai}$ and $\mathrm{Bi}$.

In Fig. 9, Ai denotes the motion between the poses $\mathrm{Ti}$ and $\mathrm{Ti}+1$. Similarly, Bi denotes the motion between $\mathrm{Ui}$ and $\mathrm{Ui}+1$.

Equation (2) can be rewritten as:

$\mathrm{A}_{\mathrm{i}} \mathrm{X}=\mathrm{XB}_{\mathrm{i}} ; \mathrm{i}=1 \ldots . .20$

and $A_{i}$ and $B_{i}$ can be calculated as

$\mathrm{A}=(\mathrm{T})^{-1} * \mathrm{~T}$

$\mathrm{Bi}=(\mathrm{Ui})^{-1} * \mathrm{U}(4)$

Where

$\mathrm{Ti}$ and $\mathrm{Ti}+1$ represent the absolute USB camera position,

expressed in the $\mathrm{CG}$ reference frame, at the $\mathrm{i}$ and $\mathrm{i}+1$ pose

$\mathrm{Ui}$ and $\mathrm{Ui}+1$ represent the absolute laparoscope position, ex- pressed in the $\mathrm{LG}$ reference frame, at $\mathrm{i}$ and $i+1$ pose.

Once $\mathrm{X}$ is derived, the LTC in (1) can be calculated as:

$\mathrm{L} \mathrm{TC}=(\mathrm{X})-1(5)$

It is important to highlight that, CGTLG matrix of (1) could be obtained by CAD measurements directly. However, considering the possible errors introduced during the fabrication of the grid supports and the inaccuracy in the grid image positioning, an alternative and more accurate method consists in deriving the transformation as in (6):

$\mathrm{CG} \mathrm{TLG}=\left(\mathrm{Ti} * \mathrm{X} *\left(\mathrm{U}_{\mathrm{i}}\right)-1\right)(6)$

After the calibration between the camera and the laparoscope, the ${ }^{\mathrm{CG}} \mathrm{TLG}$ matrix can be indeed

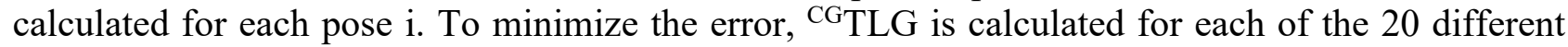
poses and the mean value is taken. 
The fourth term of (1), ${ }^{\mathrm{LG}} \mathrm{TE}$, is derived by a least-squares method [37]. In particular, the positions of three corners of the LG grid were digitized with the Aurora pointer. Then, LGTE is determined knowing the pose of these points in both the reference systems E (Aurora) and LG. Please note that the LG only serves to calibrate the system offline, thus it can be removed from the set-up during the training session to clear the surgical workspace.

The transformation CTCG $(\mathrm{t})$, which represents the extrinsic parameters of the USB camera, is calculated in real time by scanning the grid CG (which needs to be always visible by the USB camera). The employed localization algorithm is based on an iterative method for solving the PnP problem, as in [38], and it is implemented in OpenCV (Open Source Computer Vision Library).

Finally, LTE (t) can be calculated in real time from (1) and used to correctly superimpose the virtual information on the real laparoscopic image. This requires the modelling of the virtual camera, which renders the virtual content, as the real laparoscope in terms of both intrinsic and extrinsic parameters (LTE (t)) obtaining a coherent alignment between virtual and real content.

3) AR Visualization: The software framework was imple- mented in $\mathrm{C}++$ inside the Microsoft Visual Studio 2010 inte- grated development environment. The application was created following the same logic of the AR software framework previ- ously developed and presented in [39]. Such software framework includes a collection of sub-modules (i.e., dynamic libraries) and every submodule is responsible for performing specific oper- ations. From a general viewpoint, the application captures the images from the USB camera and from the laparoscope and co- herently blend the virtual content with the laparoscopic image based on a real-time estimation of the pose of the USB camera with respect to CG (CTCG (t)), and using the data coming from the EM tracker. The software framework is built upon two open- source libraries, OpenCV 3.0 and VTK 7.1 (The Visualization Toolkit). The first is a computer vision and machine learning software library (www.opencv.org); it is used to implement the calibration process. The second is a 3D computer graphics, im- age processing, and visualization library (www.vtk.org) and it is used to render the virtual scene.

The software framework is highly configurable, and it was already applied in two studies featuring the use of head-mounted displays [40], [41].

The virtual content includes the virtual models of CY-CD and CD-RHD tracts for biliary tree and CA-PHA and PHA-

a cubic Be'zier curve, commonly used for shape reconstruction in surgical applications [42]-[44] using the positional and di- rectional information of the sensors as extensively described in [22].

\section{E. Evaluation of AR Simulator}

The accuracy of AR visualization, the simulator realism, and the hardware robustness have been evaluated according to the methods described below. All tests were performed using the arterial and biliary tree standard configuration.

1) Accuracy of AR Visualization: The misalignment be- tween virtual structures and real ones gives a measure of the visualization accuracy and it is known as target visualization error (TVE) [45]. In particular, the TVE2D is the error on the image plan expressed in pixel, whereas the TVE3D is an esti- mation of the error in space expressed in mm. The TVE3D is computed knowing the laparoscope intrinsic parameters and the distance from the real points [45], [46].

During the tests, the laparoscope was placed at 12 distinct positions, varying the distance and relative orientations between laparoscope and target structures (range of distances between $90 \mathrm{~mm}$ and $170 \mathrm{~mm}$ ). The experiments were performed using the set-up described in section $\mathrm{C}$ without covering the target structures with the connective tissue; at the beginning of each experiment the position of the laparoscope was fixed.

For each position, two AR frames were acquired. The first frame recorded the target anatomy when the liver was lifted. The second recorded the anatomy with the gallbladder in lateral traction. Each AR frame was segmented to extract the real and virtual tracts of CY-CD and CD-RHD for the 
biliary tree and CA-PHA and PHA-RHA for the arterial tree. We used the Im- age Segmentation toolbox of MATLAB [47]. Then, the center- line was extracted for each segmented tract, using an algorithm based on mathematical morphology. The offset between the real and virtual centerlines of the same tract was estimated in pixel (TVE2D ); as shown in Fig. 10. The TVE3D was estimated as in

(7) by knowing: the TVE2D, the intrinsic laparoscope parame- ters and the distance between the laparoscope and target [21], [45], [46].

$\sim$ TV E2D Zc

RHA tracts for arterial tree. Virtual tracts are approximated by

$\mathrm{TVE} 3 \mathrm{D}=\mathrm{K} \quad \mathrm{f}(7)$
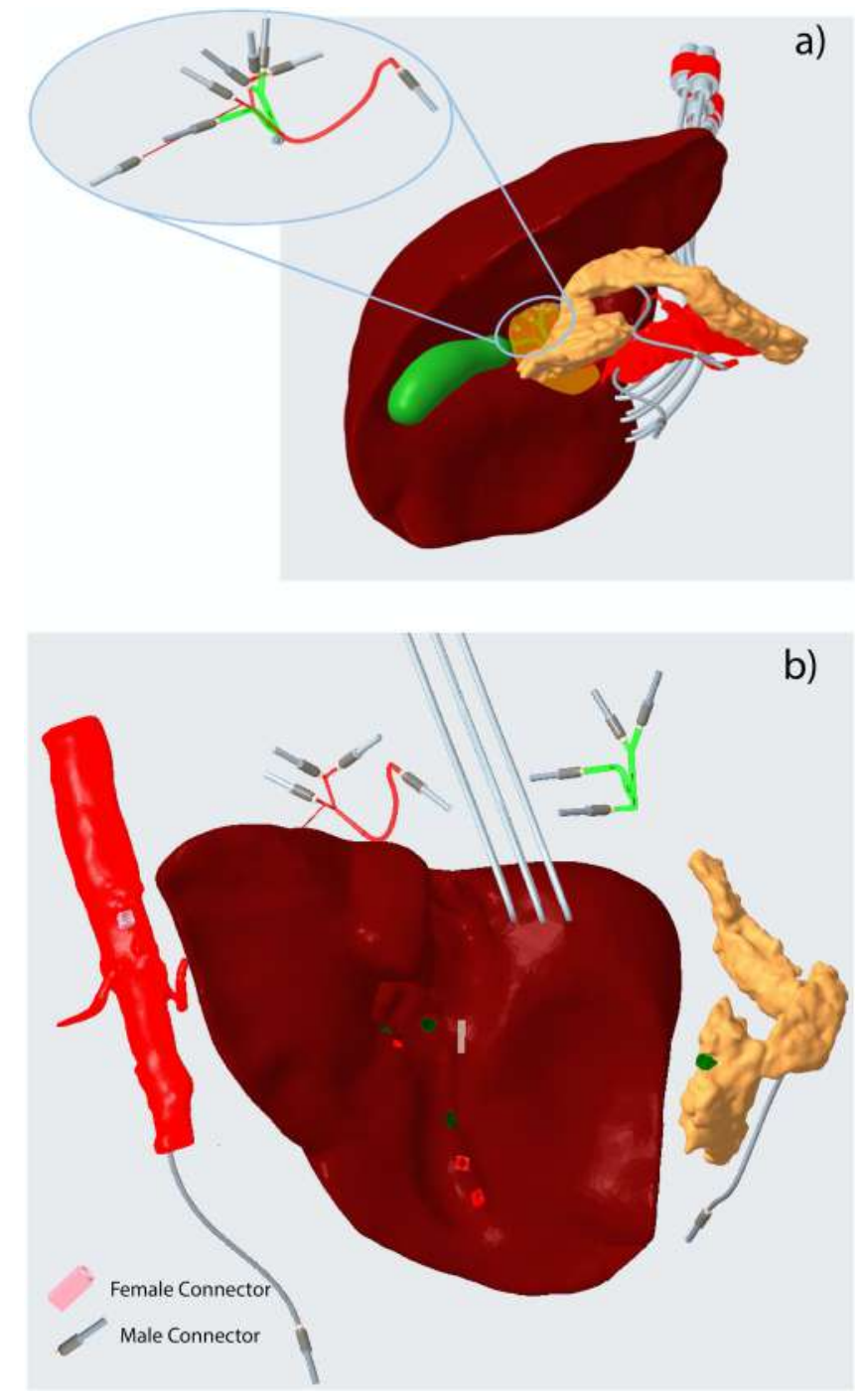

Fig. 5. Assembly and exploded view of the 3D models of the simulator:

a) Assembly view of the 3D models of the simulator; the zoomed detail shows the AT and BT not clearly visible because covered by connective tissue in whole assembly b) Exploded view of the 3D models of the simulator to show the connection strategy. 


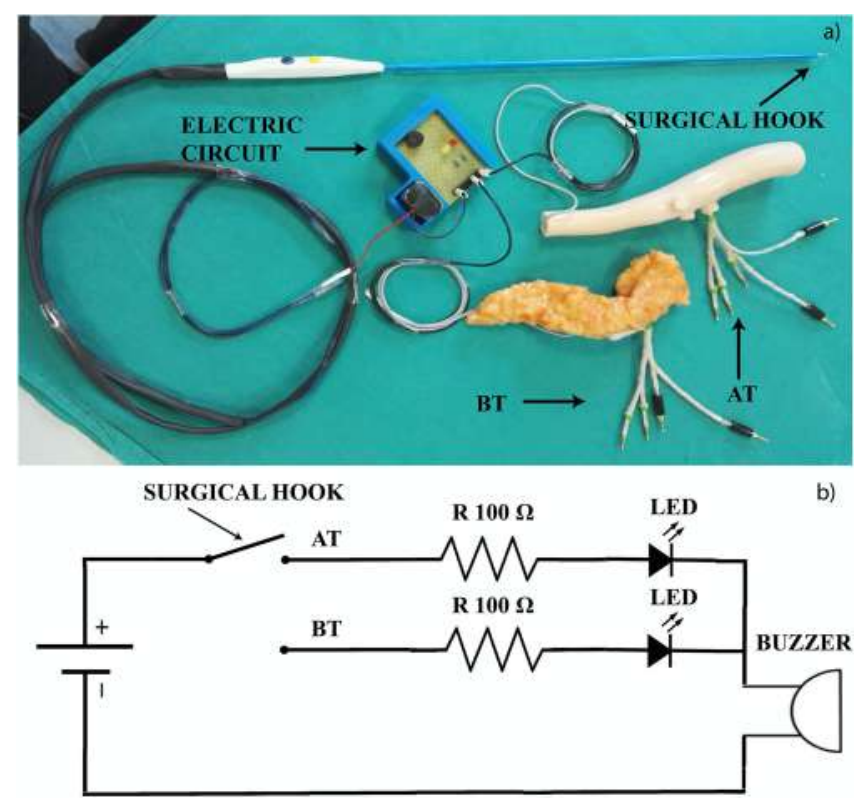

Fig. 6. Circuit for acoustic functionality: a) Simple electric circuit sig- naling the damages to AT and/or BT walls b) Equivalent electric scheme.

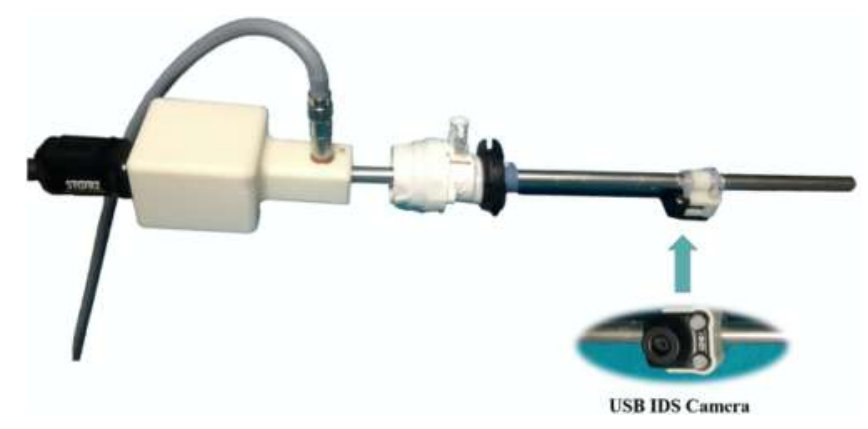

Fig. 7. Laparoscope equipped with the block and the USB camera.

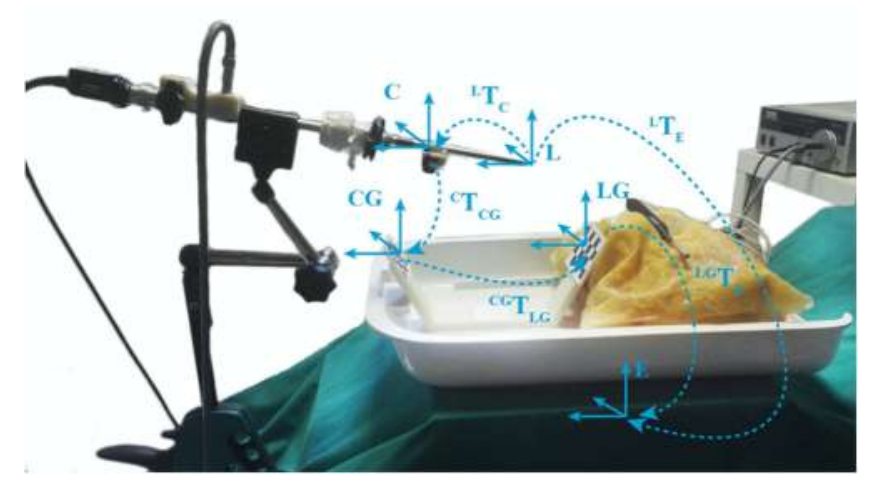

Fig. 8. Transformation (represented by dashed line) and Coordinate frames. 


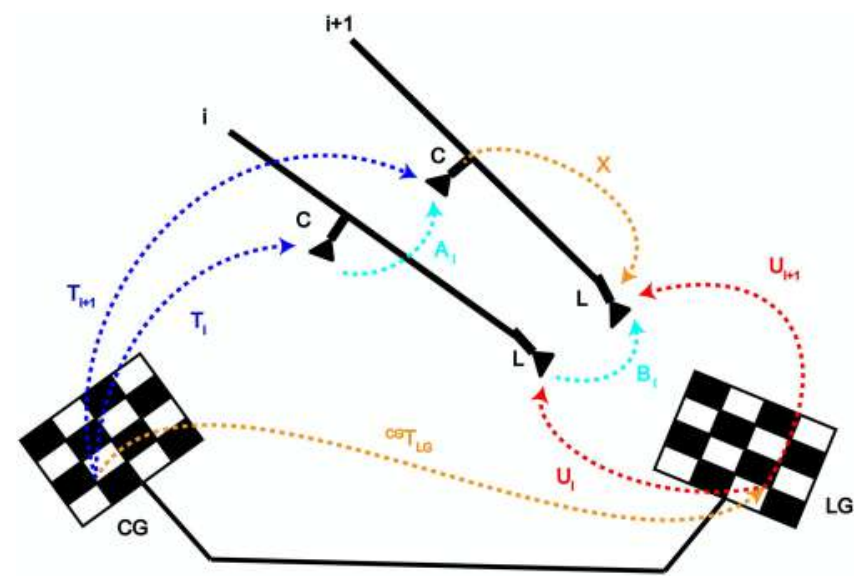

Fig. 9. Set-up of hand-eye calibration.

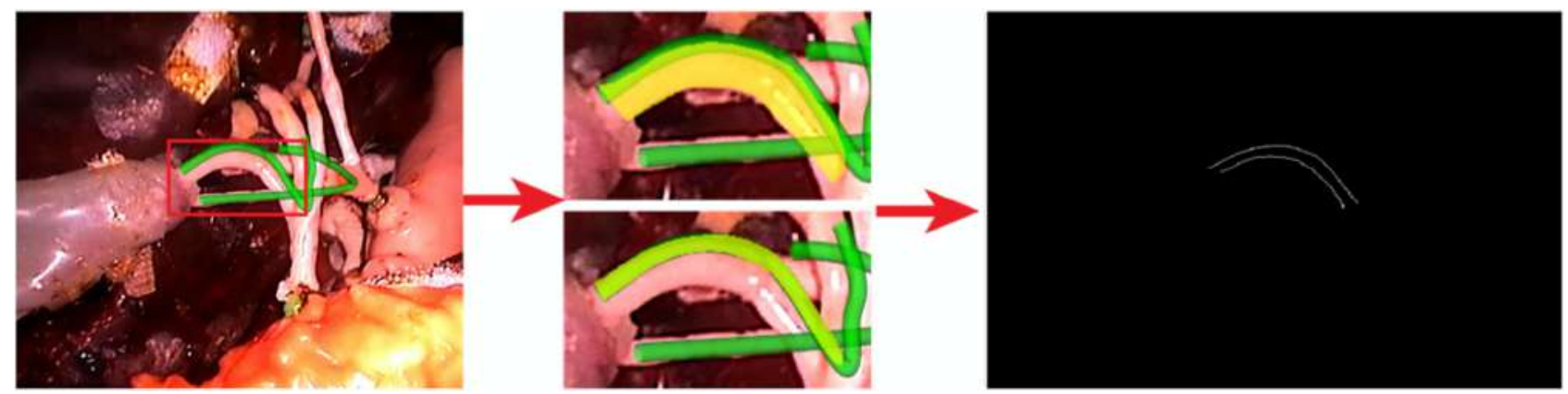

Fig. 10. Evaluation of TVE2D : a) An AR frame acquired by laparoscope. b) Segmentation of the real and the corresponding virtual CY-CD tract,

c) Extraction and comparison of two centerlines.

Where $Z_{C}$ is the estimated working distance $(\mathrm{mm})$, f repre- sents the focal length $(\mathrm{mm}), \mathrm{K}$ is the scaling factor of image sensor (number of pixels per unit of length).

In our case, the product $\mathrm{K} \mathrm{f}$ was estimated from the intrinsic parameters of the laparoscope and corresponds to 915 pixels.

TVE3D was evaluated for each of the 24 configurations, and the mean, maximum value, and standard deviation were calcu- lated for each tract. Finally, the measured TVE3D were grouped according to the diameter of tracts:

- $\quad$ Group 1: diameters from $4 \mathrm{~mm}$ to $4.5 \mathrm{~mm}$; CY-CD, CD- RHD and PHA-RHA tracts.

- $\quad$ Group 2: diameter lesser than $2 \mathrm{~mm}$; CA-PHA tract.

2) Evaluation of Simulator Realism and its Robustness:

Preliminary tests were performed to:

. assess the level of realism of the connective tissue which were never tested in our previous works.

. verify the robustness of the simulator hardware, with a focus on the connections between each simulator compo- nent.

The following surgical tools were employed for the tests: the laparoscope, laparoscopic grasping forceps and surgical hook. Ten general surgeons from the Cisanello University Hospital in Pisa were recruited in this study. 
In the experiment, the surgeons were asked to perform the identification of the cystic artery and duct: this task requires the dissection with the surgical hook of the connective tissue and the exposition of the cystic structures. During each trial, the operating surgeon performed the task while an assistant was asked to hold and direct the laparoscope. At this stage of the study, the AR visualization modality was activated at the beginning of the trial to aid the user during the recognition of the implemented anatomical variants and stopped as soon as the surgeons started to perform the dissection task.

At the end of the procedure, each surgeon was asked to com- plete a brief questionnaire comprising 6 items to assess the real- ism of the synthetic connective tissue, the usefulness of acoustic and AR functionalities. Concerning AR functionality, partici- pants were also asked to indicate in which phase of dissection task it was more useful to activate the AR functionality (before/ during/ after/ or before and after the dissection of connective tissue).

The questionnaire is based on a five-point Likert scale $(1=$ strongly disagree to 5 strongly agree), see Table IV.

Statistical analysis of data was performed using the SPSS Statistics Base 22 software. The central tendencies of responses to a single Likert item were summarized by using median, with dispersion measured by interquartile range. The Wilcoxon signed-ranks test was used to determine the significance of the responses to each item evaluating if the operators were significantly more likely to agree or disagree with each of the statements. A p-value $<0.05$ was considered statistically significant.

The sensorized structures were dissembled and reassem- bled and the connective tissue was substituted after each trial.

The robustness of the simulator hardware was evaluated by verifying the potential presence of evident damages to the reusable simulator components and their connections.

Fig. 11 shows the two surgeons performing the identification of cystic artery and duct during the trail.

TABLE IV था

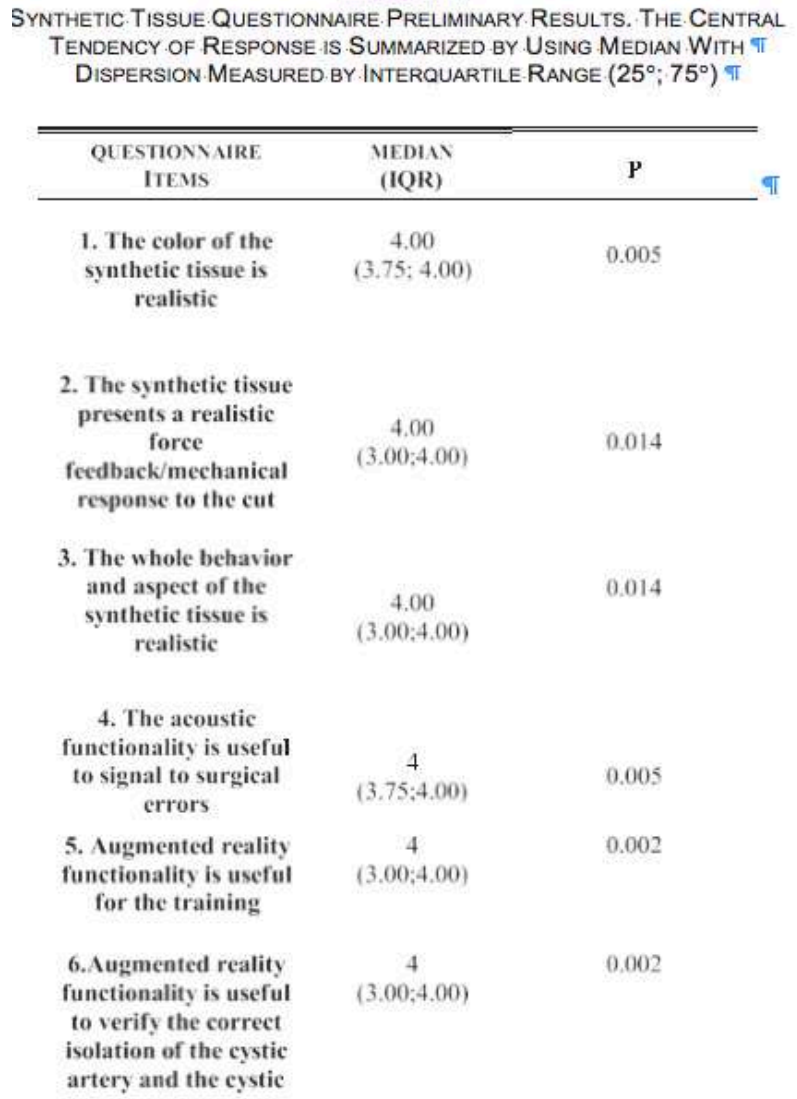




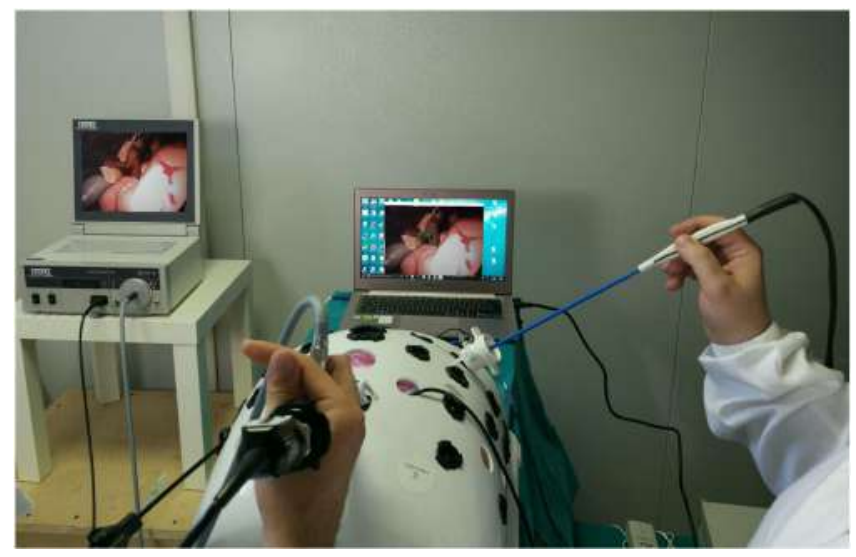

Fig. 11. Experimental test: two expert surgeons before performing the surgical test. A surgeon manages the laparoscope, while the operating surgeon holds a surgical hook (blue shaft).

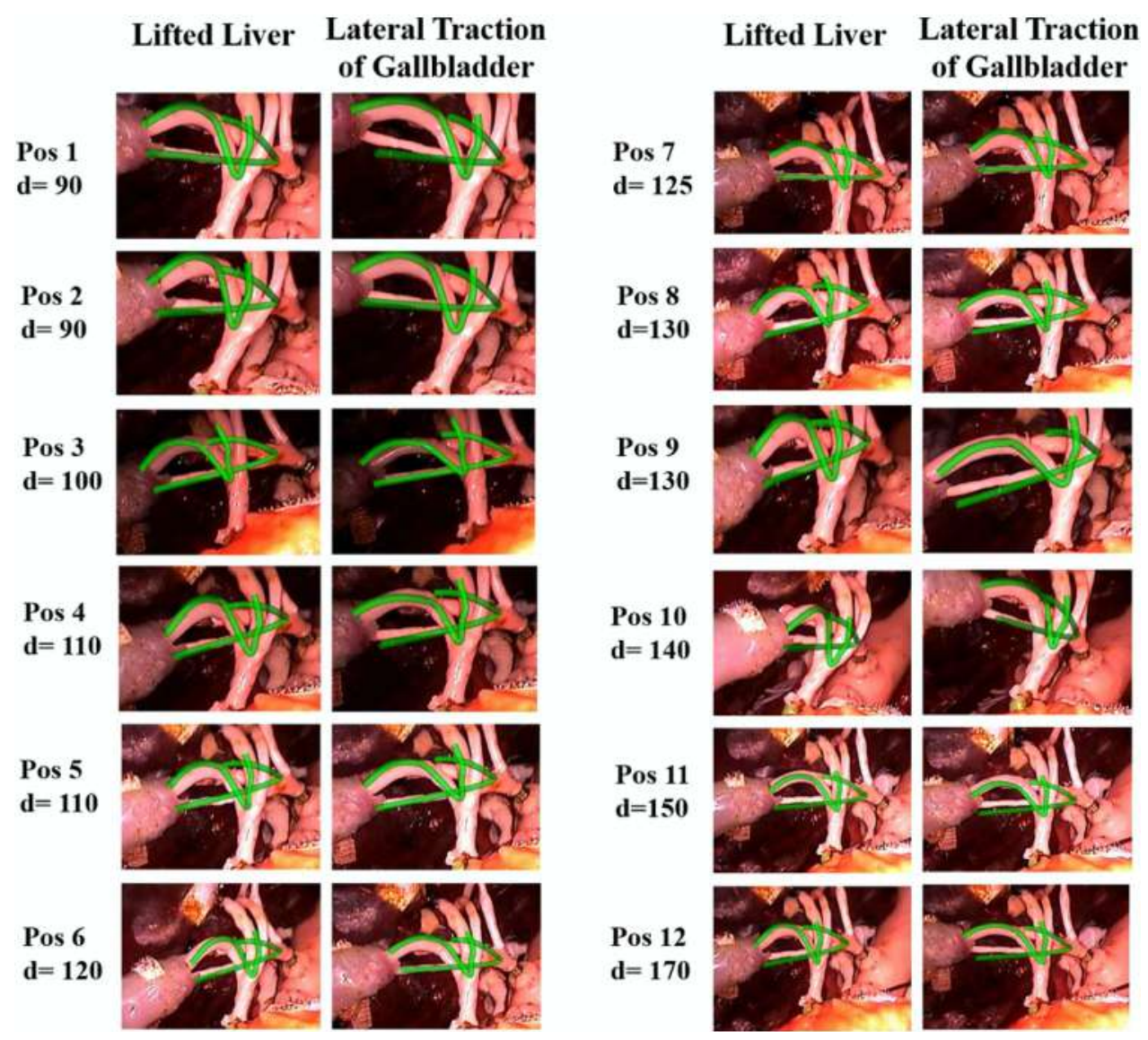

Fig. 12. AR frames for each one of the twelve positions of laparoscope in both configurations: lifted liver and gallbladder in lateral traction. The virtual tubes mimic real tracts of AT and BT, $\mathrm{d}$ is the distance between target and laparoscope expressed in $\mathrm{mm}$.

\section{RESULTS}

A. Accuracy of AR Visualization

The experimental results demonstrate that the accuracy in AR visualization is adequate for training purposes as qualitatively shown in Fig. 12.

Fig. 13 shows the mean error, maximum error and standard deviation of the TVE3D calculated for each tract. Moreover, Fig. 14 shows the relation between the TVE3D and the distance between the laparoscope and the target: the latter shows that there is not a correlation between the error and the distance. 
The TVE3D of the Group 1 (CY-CD, CD-RHD and PHA-RHA tracts) is $1.16 \pm 0.49 \mathrm{~mm}$, while for the Group 2 (CA-PHA tract) is $0.73 \pm 0.36 \mathrm{~mm}$.

B. Evaluation of Simulator Realism and Its Robustness

The surgeons positively evaluate the realism of the connective tissue and, the usefulness of both acoustic functionality and AR scene for the training.: a median score of 4 was obtained for all the items as showed by Table IV.

The surgeons asserted that the AR visualization modality is useful both before performing the dissection of the connective tissue and to verify the correct isolation of target structures after dissection.

Moreover, no damages to the simulator components and their connections were detected after the trials.

Each surgeon tried to penetrate with the surgical hook the silicone cover of the sensorized structures to test out that the acoustic functionality was able to report the occurred damage.
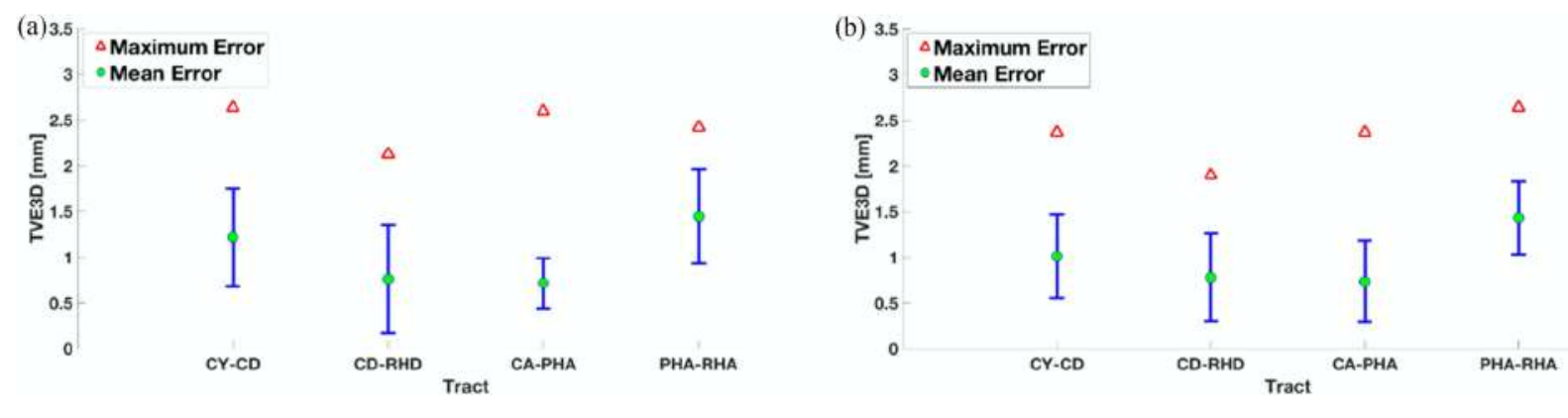

Fig. 13. AR alignment accuracy, measured as TVE3D, over each one of the four tracts for all laparoscope positions in corresponding to: a) lifted liver; b) gallbladder in lateral traction. The graph reports mean error, maximum error and standard deviation.
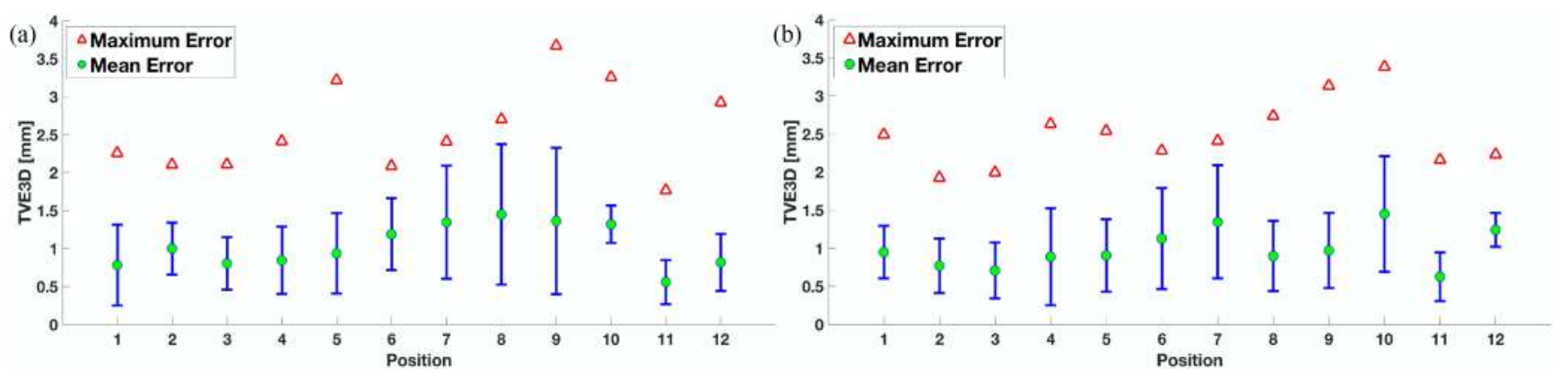

Fig. 14. AR alignment accuracy, measured as TVE3D, over each one of 12 positions of laparoscope for four tracts in corresponding to: a) lifted liver; b) gallbladder in lateral traction. The graph reports mean error, maximum error and standard deviation. 


\section{DISCUSSION}

The obtained results confirm the feasibility of the proposed strategy to track the laparoscope and the Calot's triangle to co- herently visualize the latter in AR mode. During the trials, the AR visualization was activated once the liver was lifted. The estimated static accuracy of AR visualization was considered adequate for the particular application. The estimated TVE3D is indeed lower than the anatomical structures radii: the CY-CD, CD-RHD and PHA-RHA tracts have a radius of $2 \mathrm{~mm}, 2.25 \mathrm{~mm}$ and $2.25 \mathrm{~mm}$ respectively versus a mean error of $1.16 \pm 0.49 \mathrm{~mm}$, while the CA-PHA tract presents a radius of $1 \mathrm{~mm}$ versus a mean error of $0.73 \pm 0.36 \mathrm{~mm}$. The obtained accuracy is mainly affected by four sources of error: the inherent accuracy of EM tracking paired with the field distortions arising from the environment; the difficulties in positioning the EM sensors at the tubular structures centerline [17]; the interpolation error when drawing each virtual tract; and the errors accumulated during the system calibration. As declared by the producer, the accuracy of Aurora Tabletop EM Field Generator is $1.2 \mathrm{~mm}$ for translation and $0.5^{\circ}$ rotational measurements: thus, we can affirm that the major contribution to the AR misalignment is related to the employed EM technology. The presence of metal- lic objects near the working volume further compromises the quality of the tracking. Therefore, the most urgent improvement will be the use of shielded and isolated EM sensor coils able to reduce electrical interference and to offer more mechanical robustness. The qualitative tests allowed us to complete the eval- uation of the simulator realism of the mannequin. The surgical tests confirmed the hardware robustness of the system after ex- ploring several strategies to connect the different anatomical components. In particular, the most fragile parts of the entire simulator were the electrical connections between the thin sen- sor wires (about $0.018 \mathrm{~mm}$ ) and the connectors which are the interface between the Calot's triangle and the rest of the system. To obtain an optimal view of the surgical site and to simplify the dissection task, these electrical connections are continuously pushed, pulled and twisted under the action of the surgical in- struments. The surgeons confirmed, the realism of the simulator in reproducing the interaction between the surgical instruments and the organs, the arteries and the biliary tree. They also con- firmed the usefulness of the modularity of the simulator capable of reproducing different anatomical variants.

The surgeons asserted that the AR visualization of the struc- tures inside the Calot's triangle at the beginning of the trial is particularly useful for beginners to aid the dissection task.

After the early stages of the learning curve, the AR visual- ization of the Calot's triangle should be turned on only in case of an occurring surgical damage to the sensorized structure. In the event of this happening, the AR visualization would allow the user to visualize the path of the structures that are covered by connective tissue.

Before to start the training session, an educational video will explain: the use of both AR simulation platform and surgi- cal laparoscopic instruments, and in addition the laparoscopic cholecystectomy tasks.

Specifically, the current simulator allows the execution of the following steps: trocar positioning, gallbladder retraction, grasping and lifting of the gallbladder infundibulum, dis- section of the peritoneum covering the Calot's triangle and exposure of the cystic artery and cystic duct; except from the application of clips on cystic duct and/or cystic artery and the gallbladder dissection from the liver bed.

The tutor can choose the anatomical variations of biliary and arterial tree to be implemented, on the bases of trainee experi- ence level. This gives the opportunity to create session's training with different complexity and it will allow the trainee to acquire both the dexterity necessary for good practice and the decision- making skills.

Each trainee will have the opportunity to repeat the exercise until the achievement of the proficiency level, starting with the simulation of simple anatomical cases with the possibility to "turn-on" the AR modality as a help for the identification task. The AR aid can be considered as an alternative to the tutor support. The simulation sessions will be administered until the procedure is correctly performed without the AR support and the objective of the desired performance is 
achieved. The latter will be evaluated trough the automatic evaluation system that extracts: the task execution time, the time spent outside the field of view camera instruments, the path length and the jerk, developed in our previous work [48].

Our study has some limitations. Firstly, the sample size is small because of the single institution pilot study.

Secondly, although the surgeons evaluate positively the real- ism of the simulator and recognize the potentiality of the AR functionality, this evaluation is subjective.

Thirdly, our study lacks an evaluation study that shows the efficiency of our simulator compared to existing simulators and to traditional surgical training method.

In the future work, we will conduct a multicenter study, with a larger sample and between groups with different level of ex- pertise (experts and novices), to:

- $\quad$ Demonstrate the simulator ability to discriminate between experts and novices providing an the clinically meaningful assessment [49].

- Compare our simulator and current methods of medical training demonstrating its efficiency.

In addition, we aim to conduct a study to compare simulation effectiveness, for example in terms of total training time to proficiency, of our system with and without the AR modality.

With the same aim a useful improvement for future develop- ments would be to add the bleeding and the flowing of the biliary liquid to the AR scene. The presented strategy could be applied to simulate surgical laparoscopic procedures involving the task of identification and isolation of other generic tubular structures, such as blood vessels, and nerves, which are not visible. For this reason and based on the surgeons' feedback, it could be useful to simulate the pulsatility of larger arteries, that is typically visible during the intervention. Concerning the pulsatility, it is worth mentioning that in a previous study [50] we already adopted the same electromagnetic tracking strategy together with a haptic wearable interface, to investigate the usefulness of tactile AR information, during simulated open surgeries [50]. This tactile functionality could be used in combination with the AR vi- sualization when simulating laparoscopic procedures involving larger vessels.

\section{CONCLUSION}

In this paper, an advanced version of an AR simulator for la- paroscopic cholecystectomy is presented. Tracking methods for localizing the laparoscope and the tubular anatomical structures are described. Moreover, a strategy to reconstruct the shape of the Calot's triangle structures is presented, as well as a specific calibration procedure that allows the laparoscope to be freely moved maintaining the geometric coherence of the AR scene. Preliminary tests show that the AR simulator satisfies all the initial specifications: good anatomical appearance, modularity, reusability, minimization of spare parts cost, robustness, ability to generate acoustic signaling of potential damages to the struc- tures and to provide AR visualization to show the position of the Calot's triangle structures.

Further studies will focus on demonstration of the effective- ness, of the validity and of the appropriateness of the simulator as a training tool for novices. This is the first example of a hy- brid AR simulator that offers a deep integration between real and virtual components.

Further, the presented strategy could be applied to simulate surgical laparoscopic procedures involving the task of identifi-cation and isolation of other generic tubular structures, such as blood vessels and nerves.

\section{REFERENCES}

[1] 2018. [Online]. Available: https://www.businesswire.com/news/home/ 20180119005435/en/Global-HealthcareMedical-Simulation-Market2017-2022-Product-Service 
[2] J. D. Kueck, H. D. Haynes, and R. H. Staunton, "Front cover," IEEE Pulse, vol. 6, no. 4, p. c1, 2015.

[3] H. R. Champion and A. G. Gallagher, "Surgical simulation-A 'good idea whose time has come'," Brit. J. Surg., vol. 90, no. 7, pp. 767-768, 2003.

[4] K. Gurusamy et al., "Systematic review of randomized controlled trials on the effectiveness of virtual reality training for laparoscopic surgery," Brit. J. Surg., vol. 95, no. 9, pp. 1088-1097, 2008.

[5] M. Graafland et al., "Systematic review of serious games for medical education and surgical skills training," Brit. J. Surg., vol. 99, no. 10, pp. 1322-1330, Oct. 2012.

[6] B. Kapralos et al., "An overview of virtual simulation and serious gam- ing for surgical education and training," Stud. Comput. Intell., vol. 536, pp. 289-306, Jan. 2014.

[7] K. Kowalewski et al., "Validation of the mobile serious game application Touch Surgery for cognitive training and assessment of laparoscopic chole- cystectomy," Surg. Endoscopy, vol. 31, no. 10, pp. 4058-4066, Oct. 2017.

[8] V. Lahanas et al., "Surgical simulation training systems: box trainers, vir- tual reality and augmented reality simulators," Int. J. Adv. Robot. Autom., vol. 1 no. 2, pp. 1-9, 2016.

[9] B. Dunkin et al., "Surgical simulation: A current review," Surgical En- doscopy, vol. 21, no. 3, pp. 357-366, Mar. 2007.

[10] R. S. Sidhu et al., "Assessing competency in surgery: Where to begin?"

Surgery, vol. 135, no. 1, pp. 6-20, 2004.

[11] P. D. Parchi et al., "Computer tomography prototyping and virtual pro- cedure simulation in difficult cases of hip replacement surgery," Surgical Technol. Int., vol. 23, pp. 228-234, 2013.

[12] S. M. B. I. Botden and J. J. Jakimowicz, "What is going on in augmented reality simulation in laparoscopic surgery?" Surgical Endoscopy., vol. 23, no. 8, pp. 1693-1700, Aug. 2009.

[13] S. M. B. I. Botden et al., "Augmented versus virtual reality laparoscopic simulation: What is the difference?” World J. Surg., vol. 31, no. 4, pp. 764- 772, Apr. 2007.

[14] S. M. B. I. Botden et al., "ProMIS augmented reality training of la- paroscopic procedures face validity," Simul. Healthcare: J. Soc. Simul. Healthcare, vol. 3, no. 2, pp. 97-102, Jun. 2008.

[15] S. M B. I Botden et al., "Suturing training in augmented reality: Gaining proficiency in suturing skills faster," Surgical Endoscopy, vol. 23, no. 9, pp. 2131-2137, Sep. 2009.

[16] G. Turini et al., "A microsoft hololens mixed reality surgical simulator for patient-specific hip arthroplasty training," in Proc. Int. Conf. Augmented Reality, Virtual Reality Computer Graphics, Otranto, Italy, Jun. 24-27, pp. 201-210.

[17] S. Lampotang et al., "Mixed simulators: Augmented physical simulators with virtual underlays," in Proc. IEEE Virtual Reality, Lake Buena Vista, FL, USA, Mar. 18-20, 2013, pp. 710.

[18] E. Z. Barsom et al., "Systematic review on the effectiveness of augmented reality applications in medical training," Surgical Endoscopy, vol. 30, no. 10, pp. 4174-4183, Oct. 2016.

[19] M. G. C. Pellen et al., "Construct validity of the ProMIS laparoscopic simulator," Surgical Endoscopy, vol. 23, no. 1, pp. 130-139, Jan. 2009.

[20] V. Lahanas et al., "A novel augmented reality simulator for skills assess- ment in minimal invasive surgery," Surgical Endoscopy, vol. 29, no. 8, pp. 2224-2234, Aug. 2015.

[21] V. Ferrari et al., "Augmented reality visualization of deformable tubular structures for surgical simulation,” Int. J. Med. Robot. Comput. Assisted Surg., vol. 12, no. 2, pp. 231-240, Jun. 2016.

[22] R. Viglialoro et al., "AR visualization of "synthetic Calot's triangle" for training in cholecystectomy," in Proc. 12th IASTED Int. Conf. Biomed. Eng., Innsbruck, Austria, Feb. 15-16, 2016, pp. 85-89.

[23] P. Joice et al., "Errors enacted during endoscopic surgery—a human reli- ability analysis," Appl. Ergonomics, vol. 29, no. 6, pp. 409-414, 1998. 
[24] H. C. Kuijpers and S. Klok, "Prevention and treatment of postoperative complications after stoma surgery," in Treatment of Postoperative Compli- cations After Digestive Surgery, M. A. Cuesta and H. J. Bonjer, London, U.K.: Springer, 2014, pp. 259-265.

[25] K. I. Chuang et al., "Does increased experience with laparoscopic chole- cystectomy yield more complex bile duct injuries?” Am. J. Surg., vol. 203, no. 4, pp. 480-487, Apr. 2012.

[26] R. Berguer et al., "Performing laparoscopic surgery is significantly more stressful for the surgeon than open surgery," Surgical Endoscopy. vol. 15, no. 10, pp. 1204-1207, Oct. 2001.

[27] P. Breedvel, "Observation, manipulation, and eye-hand coordination prob- lems in minimally invasive surgery," in Proc. Conf. Human Decision Mak- ing Manual Control, Kassel, Germany, Dec. 9-11, 1997, pp. 219-223.

[28] W. Breedvel, "Eye-hand coordination in laparoscopy - an overview of ex- periments and supporting aids," Minimally Invasive Therapy Allied Tech- nol., vol. 10, no. 3, pp. 155-162, Jan. 2001.

[29] R. Berguer, "Ergonomic problems associated with laparoscopic surgery,"

Surgical Endoscopy, vol. 13, no. 5, pp. 466-468, May 1999.

[30] R. Kneebone, "Simulation in surgical training: educational issues and practical implications," Med. Educ., vol. 37, no. 3, pp. 267-277, Mar. 2003.

[31] R. M. Viglialoro et al., "Augmented reality simulator for laparoscopic cholecystectomy training," in Proc. Augmented Virtual Reality: 1st Int. Conf., Lecce, Italy, Sept. 17-20, 2014, pp. $428-433$.

[32] S. Condino et al., "How to build patient-specific synthetic abdominal anatomies. An innovative approach from physical toward hybrid surgical simulators," Int. J. Med. Robot. Comput. Assisted Surg., vol. 7, no. 2, pp. 202-213, Jun, 2011.

[33] A. Pacioni et al., "Patient-specific ultrasound liver phantom: materials and fabrication method," Int. J. Comput. Assisted Radiology Surg., vol. 10, no. 7, pp. 1065-1075, Jul, 2015.

[34] S. Abdalla et al., "Calot's triangle," Clin. Anatomy, vol. 26, no. 4, pp. 493- 501, May 2013.

[35] Z. Zhang, "A flexible new technique for camera calibration," IEEE Trans. Pattern Anal. Mach. Intell., vol. 22, no. 11, pp. 1330-1334, Nov. 2000.

[36] F. C. Park and B. J. Martin, "Robot sensor calibration: solving AX $=\mathrm{XB}$ on the Euclidean group," IEEE Trans. Robot. Autom., vol. 10, no. 5, pp. 717-721, Oct. 1994.

[37] K. S. Arun et al., "Least-squares fitting of two 3-D point sets," IEEE Trans. Pattern Anal. Mach. Intell., vol. PAMI-9, no. 5, pp. 698-700, Sep.1987

[38] F. Cutolo et al., "Robust and accurate algorithm for wearable stereo- scopic augmented reality with three indistinguishable markers," Electron- ics, vol. 5, no. 3, Sep. 2016, Art. no. 59.

[39] F. Cutolo et al., "Configurable software framework for 2D/3D video see-through displays in medical applications," in Proc. Augmented Virtual Reality: 1st Int. Conf., Lecce, Italy, Jun. 15-18, 2016, pp. 30-42.

[40] F. Cutolo et al., "Perspective preserving solution for quasi-orthoscopic video see-through HMDs," Technologies, vol. 6, Jan. 2018, Art. no. 9.

[41] F. Cutolo et al., "Hybrid video/optical see-through HMD," in Proc. IEEE Int. Symp. Mixed Augmented Reality, Nantes, France, Oct. 9-13, 2017, pp. 52-57.

[42] X. Li et al., "Endoscopes shape reconstruction based on electromagnetic localization and curve fitting," in Proc. IEEE Int. Conf. Robot. Biomimet- ics, Guangzhou, China, Dec. 11-14, 2012, pp. 819-824.

[43] Y. Cai et al., "Constructive algorithms of vascular network modeling for training of minimally invasive catheterization procedure," Adv. Eng. Software, vol. 34, no. 7, pp. 439-450, Jul. 2003.

[44] S. Shuang et al., "Electromagnetic positioning for tip tracking and shape sensing of flexible robots," IEEE Sensors J., vol. 15, no. 8, pp. 4565-4575, Aug. 2015.

[45] M. Mu"ller et al., "Mobile augmented reality for computer-assisted per- cutaneous nephrolithotomy," Int. J. Comput. Assisted Radiology Surg., vol. 8, no. 4, pp. 663-675, Jul. 2013. 
[46] M. Baumhauer et al., "Soft tissue navigation for laparoscopic partial nephrectomy," Int. J. Comput. Assisted Radiology Surg., vol. 3, no. 3, May 2008, Art. no. 307.

[47] B. K. Jang and R. T. Chin, "Analysis of thinning algorithms using math- ematical morphology," IEEE Trans. Pattern Anal. Mach. Intell., vol. 12, no. 6, pp. 541-551, Jun. 1990.

[48] V. Mamone et al., "Robust laparoscopic instruments tracking using colored strips," in Proc. Int. Conf. Augmented Reality, Virtual Reality Comput. Graphics, Ugento, Italy, Jun. 12-15, 2017, pp. 129-143.

[49] A. Moglia et al., "A systematic review of virtual reality simulators for robot-assisted surgery," Eur. Urology, vol. 69, no. 6, pp. 1065-1080, Jun. 2016.

[50] S. Condino et al., "Tactile augmented reality for arteries," in Proc. Int. Conf. Med. Imaging Virtual Reality, Bern, Switzerland, Aug. 24-26, 2016, pp. 186-197. 